PHYSICAL REVIEW D 93, 019901(E) (2016)

\title{
Erratum: Pseudoscalar meson and charmed baryon scattering lengths [Phys. Rev. D 86, 034009 (2012)]
}

Zhan-Wei Liu and Shi-Lin Zhu

(Received 23 December 2015; published 12 January 2016)

DOI: 10.1103/PhysRevD.93.019901

The low energy constants $\bar{c}_{0}, c_{0}, c_{3}$, and $c_{4}$ in Eq. (B6) in the Appendix should be replaced with the following expressions:

$$
\begin{aligned}
& \bar{c}_{0}=\frac{1}{2} \alpha_{0}-\frac{1}{36} \alpha_{1}+\frac{1}{36} \alpha_{2}=-0.32 \mathrm{GeV}^{-1}, \quad c_{0}=\alpha_{0}-\frac{1}{6} \alpha_{1}-\frac{1}{6} \alpha_{2}=-0.61 \mathrm{GeV}^{-1}, \\
& c_{3}=\frac{1}{2} \alpha_{6}+\frac{1}{2} \alpha_{7}=-0.84 \mathrm{GeV}^{-1}, \quad c_{4}=\alpha_{3}=\frac{\alpha^{\prime}}{4 \pi f} .
\end{aligned}
$$

Accordingly, Eq. (19) and the numbers in Tables I, II, and III should also be updated. The adjustment lies within the theoretical error of the LEC estimation, and our main conclusions do not change. However, one should remove $T_{\eta \Lambda_{c}}^{(0)}, T_{\pi \Sigma_{c}}^{(1)}$, and $T_{\pi \Sigma_{c}^{*}}^{(1)}$ from the convergent $T$ matrices and add $T_{\bar{K} \Sigma_{c}}^{(3 / 2)}$ and $T_{\bar{K} \Sigma_{c}^{*}}^{(3 / 2)}$ in the third paragraph in Sec IV.

There were typos in Eqs. (B2), (B5), (B6), and (19). "GeV" should be replaced with "GeV ${ }^{-1}$," and $T_{123}$ and $T_{132}$ should read as

$$
T_{123}=\frac{1}{2} \Sigma^{0}-\frac{1}{2 \sqrt{3}} \Lambda, \quad T_{132}=-\frac{1}{\sqrt{3}} \Lambda .
$$

